\title{
Difficulties in the diagnosis of soft tissue sarcomas
}

\author{
R. Jha \\ Department of Pathology, Institute of Medicine, Tribhuvan University Teaching Hospital Kathmandu; Nepal
}

Correspondence to: Dr Runa Jha, Department of Pathology, TUTH, Maharajgung , Kathmandu, Nepal. Po Box No. 3578

Email: runa75jha@gmail.com

\begin{abstract}
Introduction: Soft tissue tumors represent diagnostic challenge to general practicing pathologists because of overlap in morphologic features. The usual approach is to diagnose by presumed cell lineage. A major utility of immunohistochemistry is to identify a tumor being mesenchymal or nonmesenchymal and once mesenchymal lineage has been confirmed, histologic sub typing according to specific lineage may be achieved with help of lineage specific markers.

Methods: The objective of this study was to analyse different types of soft tissue malignancies according to their type, site and age distribution, discuss the difficulties faced during morphologic examination and review the literature for role of ancillary techniques, particularly immunohistochemistry in diagnosing soft tissue sarcomas. All soft tissue malignancies reported from Tribhuvan University Teaching Hospital in three years period from April 2008 to March 2010 were included in the study.

Results: Total 87 soft tissue malignancies were reported during study period. Most of these patients (58.6\%) were male and the rest were female. These malignancies were found in all age groups. Youngest patient was newborn who was born with tongue mass and biopsy was performed on 13th day of life. However maximum numbers of tumors (41.4\%) were seen in first two decades of life. Head and neck was the most common site . Rhabdomyosarcoma was the most common diagnosis. In 20 cases (23\%) a definite opinion was not possible and vague terms like sarcoma, small round cell tumor, spindle cell malignancy, vascular tumor "likely" were used. Immunohistochemistry was advised in these cases.

Conclusions: Only light microscopic examination of hematoxylin and eosin stained slide not sufficient at all times because there is morphologic overlap of soft tissue sarcomas with each other and with carcinomas and melanomas. Though immunohistochemistry is not a substitute for skilled interpretation of routinely stained slides but prove helpful to reach a more definite diagnosis.
\end{abstract}

Keywords: Immunohistichemistry, sarcoma, soft tissue

\section{Introduction}

Soft tissue tumors represent diagnostic challenge to general practicing pathologists because of overlap in morphologic features. The usual approach is to diagnose by presumed cell lineage. The World Health Organization classification, divides tumors into adipocytic, fibroblastic/myofibroblastic, so-called fibrohistiocytic, smooth muscle, pericytic, skeletal muscle, vascular, chondro-osseous, and lastly "of uncertain differentiation". ${ }^{1}$
Immunohistochemistry helps to identify a tumor being mesenchymal or non mesenchymal and once mesenchymal lineage has been confirmed, histologic sub typing according to specific lineage may be achieved with help of lineage specific markers. ${ }^{2}$

The objective of this study was to analyse different types of soft tissue malignancies according to their type, site and age distribution, discuss the difficulties faced during morphologic examination and review the literature for role 
of ancillary techniques, particularly immunohistochemistry in diagnosing soft tissue sarcomas.

\section{Methods}

A retrospective observational study of soft tissue malignancies reported from Tribhuvan University Teaching Hospital in three years period from April 2008 to March 2010 was conducted. This included all the cases where soft tissue malignancy was the only diagnosis or was one of the differential diagnoses on histopathology. Same patient with biopsies performed on different occasions were considered as one case.

\section{Results}

Out of 18,508 biopsies examined during the study period, 87 were soft tissue malignancies. There were 51 males (58.6\%) and the rest were female. These malignancies were found in all age groups. Youngest patient was newborn who was born with tongue mass and biopsy was performed on $13^{\text {th }}$ day of life. Oldest patient was 85 years. However maximum numbers of tumors ( 36 out of 87 i.e. $41.4 \%$ ) were seen in first two decades of life. The site of tumor in this study was divided into 6 categories (Table 1). In head and neck group, all tumors arising in the area was grouped together irrespective of the location of tumor. Tumors grouped to be arising from trunk included all superficial tumors located in this region. Deep tumors arising from soft tissue and visceras in retroperitoneum were placed in separate group where as deep tumors located in mediastinum, abdomen (other than retroperitoneum) and in reproductive tract were placed in "others" category. The upper and lower limb category included all tumors arising in these regions. Following this categorization, head and neck was the most common site involved closely followed by trunk and lower limb (Table 1).

Table 1: Site distribution of sarcomas in different age groups

\begin{tabular}{|c|c|c|c|c|c|c|c|}
\hline Lower limb & Trunk & & $\mathrm{um}$ & Others & Total & & \\
\hline$<1$ & 1 & & 1 & 1 & 1 & & 4 \\
\hline $1-10$ & 11 & & & 1 & 2 & 1 & 15 \\
\hline $11-20$ & 5 & & 6 & 4 & & 2 & 17 \\
\hline $21-30$ & 1 & & 3 & 1 & & 2 & 7 \\
\hline $31-40$ & 1 & & 3 & 1 & 1 & 2 & 8 \\
\hline $41-50$ & & 1 & 1 & 3 & 1 & 1 & 7 \\
\hline $51-60$ & & 1 & 3 & 4 & 2 & 2 & 12 \\
\hline $61-70$ & 2 & 1 & & 2 & 1 & 3 & 9 \\
\hline$>70$ & 1 & & 1 & 4 & 1 & 1 & 8 \\
\hline Total & 22 & 3 & 18 & 21 & 9 & 14 & 87 \\
\hline
\end{tabular}

Immunohistochemistry to confirm diagnosis was advised in all these doubtful cases.

Table 2: Different types of soft tissue sarcomas

$\begin{array}{ll}\text { Diagnosis } & \text { Number (\%) } \\ \text { Embryonal rhabdomyosarcoma } & 9(10.3) \\ \text { Alveolar rhabdomyosarcoma } & 5(5.7) \\ \text { Malignant GIST } & 7(8.0) \\ \text { Dermatofibrosarcoma protruberance } & 6(6.9) \\ \text { Synovial sarcoma } & 5(5.7) \\ \text { Ewing sarcoma } & 5(5.7) \\ \text { Leiomyosarcoma } & 4(4.6) \\ \text { MPNST } & 4(4.6) \\ \text { osteosarcoma } & 4(4.6) \\ \text { Dedifferentiated liposarcoma } & 4(4.6) \\ \text { MFH } & 3(3.4) \\ \text { Angiosarcoma } & 3(3.4) \\ \text { Chondrosarcoma } & 3(3.4) \\ \text { Clear cell sarcoma } & 3(3.4) \\ \text { Alveolar soft part sarcoma } & 1(1.1) \\ \text { Sarcoma } & 7(8.0) \\ \text { Small round cell tumor } & 5(5.7) \\ \text { Spindle cell malignancy } & 4(4.6) \\ \text { Malignant vascular tumor likely } & 2(2.3) \\ \text { Pleomorphic sarcoma } & 2(2.3) \\ \text { Total } & \mathbf{8 7}(\mathbf{1 0 0 . 0 )}\end{array}$

\section{Discussion}

The immunohistochemical identification of various cell and tissue markers specific for certain avenues of cell differentiation has permitted more accurate diagnosis and classification of these tumors. ${ }^{3}$ Rhabdomyosarcoma are group of tumors with skeletal muscle differentiation. These tumors are divided into 3 main biologically distinct categories like embryonal rhabdomyosarcoma (ERMS), 
alveolar rhabdomyosarcoma (ARMS), and pleomorphic rhabdomyosarcoma (PRMS). ${ }^{1}$ The role of IHC in these tumors is mainly to confirm their skeletal muscle lineage, which may not be apparent on H\&E morphology. The two overall most useful markers in RMS diagnosis are desmin and myogenin. Desmin is highly sensitive for all tumors with skeletal differentiation but somewhat nonspecific because it may also stain smooth muscle cells and occasionally even myofibroblasts. MyoD1 and myogenin are very specific for skeletal muscle. ${ }^{2}$

In 5 cases, the diagnosis was malignant small round cell tumor and in 4 cases, our diagnosis was spindle cell malignancy.

Morphologic differential diagnosis of malignant small round cell tumors include Ewing's sarcoma/PNET, neuroblastoma, rhabdomyosarcoma, desmoplastic small round-cell tumor, lymphoma, leukemia, small-cell osteosarcoma, small-cell carcinoma (either undifferentiated or neuroendocrine), olfactory neuroblastoma, cutaneous neuroendocrine carcinoma, (Merkel-cell carcinoma), small-cell melanoma and mesenchymal chondrosarcoma. ${ }^{4}$

Cytoplasm of Ewing sarcoma contains periodic acid Schiff (PAS) positive glycogen and CD 99 is expressed in almost all cases in characteristic membranous fashion. There is no specific phenotype for small cell osteosarcoma. But these small cells are associated with osteoid production. ${ }^{1}$

The list of differential diagnosis for spindle cell malignancy is long some being leiomyosarcoma, gastrointestinal stromal tumor (GIST), malignant peripheral nerve sheath tumor(MPNST), monophasic synovial sarcomas, spindle cell melanomas, sarcomatoid carcinomas and so on. A clinical correlation with site of lesion and other findings like immunohistochemistry may help to reach a more definite opinion. Leiomyoma and leiomyosarcomas(LMS) are generally strongly and uniformly positive for smooth muscle actin (SMA) and HHF35. 70 to $80 \%$ of leiomyosarcomas are desmin positive. To define a poorly differentiated spindle cell sarcoma as LMS, at least 2 of 3 muscle markers (using for example SMA/desmin/HHF35 or SMA/desmin/calponin) should be positive. ${ }^{2,5}$

Before the discovery of the KIT receptor kinase gene mutations and associated expression of Kit protein detected by IHC, many GISTs were classified as smooth muscle tumors. Kit (CD117) positivity is seen in the great majority of GISTs $(>95 \%)$ Other spindle cell malignancies that resemble GIST are c Kit negative. ${ }^{6,7}$

It is often difficult to differentiate dermatofibroma from dermatofibrosarcoma protruberance (DFS) because of overlapping morphology. DFS is factor XIIIa negative and CD 34 positive whereas it is opposite in dermatofibroma. Also dermatofibroma is positive for HMGA 1 and HMG 2 and DFS is negative for this two. ${ }^{7}$

In 7 cases the diagnosis was only sarcoma with different differential diagnosis being myxoid chondrosarcoma, liposarcoma, malignant fibrous histiocytoma (MFH), leiomyosarcoma and pleomorphic rhabdomyosarcoma. Pleomorphic sarcomas may be mimicked by sarcomatoid carcinomas and melanomas as well. The initial battery of IHC markers should always include a broad keratin (pankeratin), S100, and vimentin. A positive requires work up to detect primary carcinoma elsewhere. Additional immunohistochemical stains, including keratin subtypes, may be used to help narrow this search. A positive S100 stain should be followed by additional melanoma markers. ${ }^{2,8}$ Immunohistochemistry is very useful in determining vascular lineage, especially in poorly differentiated angiosarcoma and vascular tumors mimicking epithelial neoplasms (epithelioid hemangioendothelioma and epithelioid angiosarcoma). Commonly used vascular markers are factor VIII, CD34, CD31, and FLI-1.,

Apart from immunohistochemistry, electron microscopy and cytogenetic analysis also help to differentiate different types of soft tissue sarcomas. ${ }^{10-13}$ Histological classification of soft tissue sarcomas can be made by light microscopy alone for those tumors with unique appearances, while a significant proportion of sarcomas require the implementation of electron microscopy or immunohistochemistry to obtain a final diagnostic classification. ${ }^{14}$

So relying upon morphology alone a definite diagnosis is not always possible. Difficulties increase when the relevant important features like age of patient, site of lesion, size of lesion and radiologic findings are not provided with the requisition form.

Another common problem with small biopsies is that the biopsied tissue might not be representative of entire lesion. Moreover sometimes the tissue removed is divided into multiple pieces and sent to different pathologists. These different pieces might be representing just one feature each of the actual lesion thus adding to the diagnostic dilemma and leading to wrong diagnosis.

\section{Conclusions}

Light microscopic examination of $\mathrm{H} / \mathrm{E}$ stained slide is not sufficient at all times because there is morphologic overlap of soft tissue sarcomas with each other and with carcinomas 
and melanomas. Good history, adequate sampling, radiologic findings, exact location narrows the diagnosis. Though immunohistochemistry is not a substitute for skilled interpretation of conventionally stained microscopic specimens, it helps to reach a more definite diagnosis.

\section{Recommendations}

The biopsy sent for evaluation should always be accompanied by adequate clinical information and relevant investigation findings. The laboratory should establish the habit of refusing to accept samples with inadequate accompanying information. Practice of breaking down the biopsied tissue into multiple pieces and sending to different pathologists should be strongly discouraged. We need to understand the limitations of conventional methods and clinico pathologic discussion should always be encouraged. Moreover development of ancillary techniques, particularly immunohistochemistry is strongly required, which is in very primitive stage in Nepal. It would always be cost effective to spend some extra money on investigations to get a more definite correct diagnosis then to spend on expensive treatments with wrong diagnosis.

\section{References}

1. Fletcher CD, Unni KK, Mertens F, editors. Pathology and genetics of tumours of soft tissue and bone. Lyon, France: IARC Press; 2002. p. 120. (World Health Organization Classification of Tumours; vol 5).

2. Heim-Hall J, Yohe SL. Application of immunohistochemistry to soft tissue neoplasms. Arch Pathol Lab Med. 2008;132(3):476-89.

3. Ordonez NG. Application of immunocytochemistry in the diagnosis of soft tissue sarcomas: a review and update. Adv Anat Pathol. 1998;5(2):67-85.

4. Devoe K, Weidner N. Immunohistochemistry of small round-cell tumors. Semin Diagn Pathol. 2000;17(3): 216-24.

5. Al-Daraji W, Husain E, Zelger BG, Zelger BA. Practical and comprehensive immunohistochemical approach to the diagnosis of superficial soft tissue tumors. Int J Clin Exp Pathol 2009;2:119-131.

6. Miettinen M, Lasota J. Gastrointestinal stromal tumors (GISTs): definition, occurrence, pathology, differential diagnosis and molecular genetics. Pol J Pathol $2003 ; 54$ : 3-24.

7. Miettinen M. Immunohistochemistry in soft tissue tumors, an update. Recent advances and controversies in soft tissue. pathology 1999;32:3.

8. Wick MR. Immunohistochemical approaches to the diagnosis of undifferentiated malignant tumors. Annals of Diagnostic Pathology 2008;12:72-84.

9. Sirgi KE, Wick MR, Swanson PE. B72.3 and CD34 immunoreactivity in malignant epithelioid soft tissue tumors. Adjuncts in the recognition of endothelial neoplasms. Am J Surg Pathol. 1993;17:179-85.

10. Kandel R, Bedard YC, Fan QH. Value of electron microscopy and immunohistochemistry in the diagnosis of soft tissue tumors. Ultrastructural Pathology 1998;22:141-6.

11. Fisher C. Comparative role of electron microscopy and immunohistochemistry in diagnosis of soft tissue tissue tumors. Histopathology 2006:48:32-41.

12. Sandberg AA, Bridge JA .Updates on cytogenetics and molecular genetics of bone and soft tissue tumors: Ewing sarcoma and peripheral primitive neuroectodermal tumors. Cancer genetics and cytogenetics. 2000;123:1-26.13. Limon J, Mrozek K, Mandahl N, Nedoszytko B, Verhest A, Rys J, et al. Cytogenetics of synovial sarcoma: presentation of ten new cases and review of the literature. Genes Chromosomes Cancer. 1991;3:33845.14. Carbone A, Gloghini A, Volpe R. The value of immunohistochemistry in the diagnosis of soft tissue sarcomas. Ann Oncol. 1992; (Suppl 2):S51-54. 